\title{
Reduced leakage currents and possible charge carriers tuning in Mg-doped $\mathrm{Ga}_{0.6} \mathrm{Fe}_{1.4} \mathrm{O}_{3}$ thin films
}

C. Lefevre, R. H. Shin, J. H. Lee, S. H. Oh, F. Roulland, A. Thomasson, E. Autissier, C. Meny, W. Jo, and N. Viart

Citation: Appl. Phys. Lett. 100, 262904 (2012); doi: 10.1063/1.4729872

View online: https://doi.org/10.1063/1.4729872

View Table of Contents: http://aip.scitation.org/toc/apl/100/26

Published by the American Institute of Physics

\section{Articles you may be interested in}

Room temperature multiferroicity in $\mathrm{Ga}_{0.6} \mathrm{Fe}_{1.4} \mathrm{O}_{3}: \mathrm{Mg}$ thin films

Journal of Applied Physics 113, 214101 (2013); 10.1063/1.4808349

Electrical, magnetic, magnetodielectric, and magnetoabsorption studies in multiferroic $\mathrm{GaFeO}_{3}$ Journal of Applied Physics 106, 123910 (2009); 10.1063/1.3271391

Room-temperature polarization switching and antiferromagnetic coupling in epitaxial $(\mathrm{Ga}, \mathrm{Fe})_{2} \mathrm{O}_{3} / \mathrm{SrRuO}_{3}$ heterostructures

Applied Physics Letters 106, 142902 (2015); 10.1063/1.4917249

Control of crystal-domain orientation in multiferroic $\mathrm{Ga}_{0.6} \mathrm{Fe}_{1.4} \mathrm{O}_{3}$ epitaxial thin films

Applied Physics Letters 110, 212905 (2017); 10.1063/1.4984211

Origin of the different multiferroism in $\mathrm{BiFeO}_{3}$ and $\mathrm{GaFeO}_{3}$

Journal of Applied Physics 113, 063905 (2013); 10.1063/1.4791586

Epitaxial thin films of multiferroic $\mathrm{GaFe}_{3}$ on conducting indium tin oxide (001) buffered yttrium-stabilized zirconia (001) by pulsed laser deposition

Applied Physics Letters 91, 202504 (2007); 10.1063/1.2813020

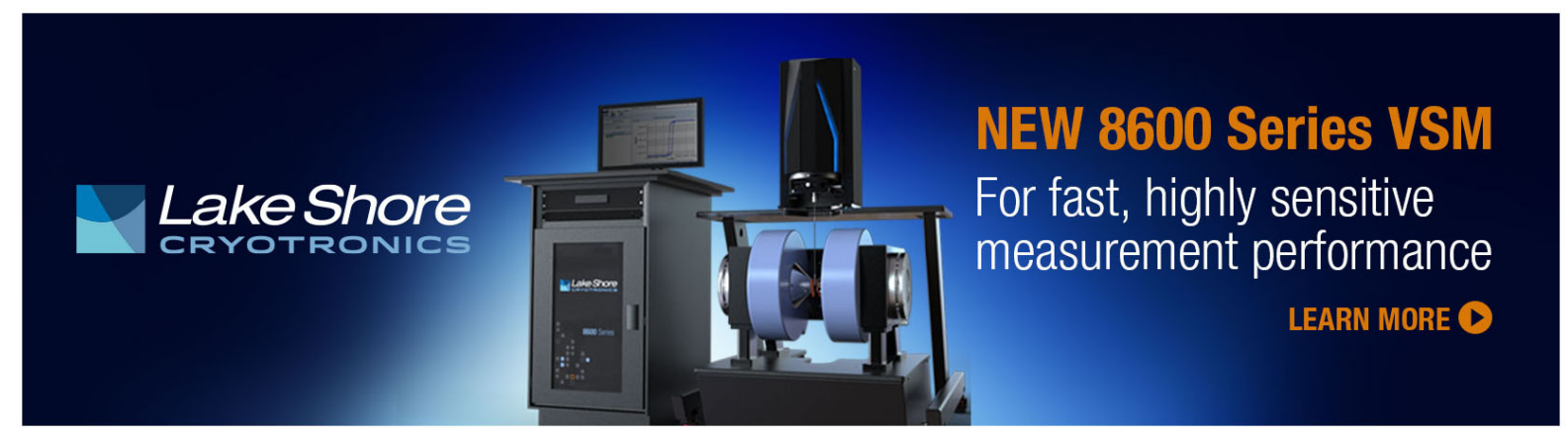




\title{
Reduced leakage currents and possible charge carriers tuning in Mg-doped $\mathrm{Ga}_{0.6} \mathrm{Fe}_{1.4} \mathrm{O}_{3}$ thin films
}

\author{
C. Lefevre, ${ }^{1, a)}$ R. H. Shin, ${ }^{2}$ J. H. Lee,${ }^{2,3}$ S. H. Oh, ${ }^{2}$ F. Roulland, ${ }^{1}$ A. Thomasson, ${ }^{1}$ \\ E. Autissier, ${ }^{1}$ C. Meny, ${ }^{1}$ W. Jo, ${ }^{2}$ and N. Viart ${ }^{1}$ \\ ${ }^{1}$ Institute of Physics and Chemistry of Materials of Strasbourg, UMR 7504 UDS-CNRS, \\ Strasbourg 67034, France \\ ${ }^{2}$ Department of Physics, Ewha Womans University, Seoul 120-750, Korea \\ ${ }^{3}$ CNRS-EWHA International Research Center, Ewha Womans University, Seoul 120-750, Korea
}

(Received 12 January 2012; accepted 30 May 2012; published online 26 June 2012)

\begin{abstract}
$\mathrm{Ga}_{0.6} \mathrm{Fe}_{1.4} \mathrm{O}_{3}$ is predicted to be magnetoelectric with non zero magnetization at room temperature. However, in thin films, electric properties are overshadowed by strong leakage currents. In this Letter, we show that $\mathrm{Mg}$ doping in $\mathrm{Ga}_{0.6} \mathrm{Fe}_{1.4} \mathrm{O}_{3}$ thin films grown by pulsed laser deposition allows decreasing the leakage current density by four orders of magnitude and might simultaneously allow tuning the carriers' nature. These results suggest the possibility to develop a new class of material exhibiting room temperature magnetization, tunable transport properties, and magnetoelectric properties. (C) 2012 American Institute of Physics. [http://dx.doi.org/10.1063/1.4729872]
\end{abstract}

\begin{abstract}
Magnetoelectric materials open up the way to many applications thanks to the possibility to control their magnetic polarization and their electric polarization with an electric field and a magnetic field, respectively. For these reasons, magnetoelectric materials receive a considerable renewal of interest for their potential use in new electronic devices. ${ }^{1-3}$ The $\mathrm{Ga}_{2-\mathrm{x}} \mathrm{Fe}_{\mathrm{x}} \mathrm{O}_{3}(0.8 \leq \mathrm{x} \leq 1.4)$ (GFO) oxides present all necessary properties to be promising candidates for such applications. ${ }^{4,5}$ These compounds crystallize in an orthorhombic structure (S.G: $P c 2_{1} n$ ) with $\mathrm{a} \sim 8.7 \AA$, $\mathrm{b} \sim 9.4 \AA$, and $\mathrm{c} \sim 5.1 \AA$. The materials are ferrimagnetic with Curie temperatures up to $350 \mathrm{~K}$ for $\mathrm{x}=1.4$. Finally, magnetoelectric effects have been observed in these materials, in bulk, by Rado. ${ }^{4}$ If the bulk properties of the compound have been well established already in the 1960s, high quality thin films, necessary for applications, have only been produced recently. ${ }^{6}$ These films show the same crystallographic and magnetic features as the bulk, but important leakage currents hinder their electric characterization. These leakage currents probably result from a substoichiometry of the films in oxygen. The resulting reduction of $\mathrm{Fe}^{3+}$ to $\mathrm{Fe}^{2+}$ in order to counterbalance the charge deficiency might decrease the electrical resistivity of GFO thin films through a hopping mechanism. These leakage currents overshadow the polarization-electric field (P-E) signal and can lead to a misinterpretation of the ferroelectric loops. ${ }^{7}$ Such behaviors have been observed in other oxide systems like $\mathrm{BiFeO}_{3}, \mathrm{YMnO}_{3}$, or $\mathrm{Ni}_{3} \mathrm{~V}_{2} \mathrm{O}_{8}$ [Ref. 8 and references therein]. The leakage currents problem needs to be thoroughly studied and solved for thin films that will be inserted in functional devices for their magnetoelectric properties. ${ }^{9}$ There has been a flurry of studies in the literature in connection with the understanding of leakage mechanisms in ferroelectric thin films. ${ }^{10-13}$ Several groups have explored the effects of doping in order to reduce the leakage currents. ${ }^{14-16}$ In this paper, we focus on the substitution of Fe with Mg. While Fe can be bi- or
\end{abstract}

a)E-mail: christophe.lefevre@ipcms.unistra.fr. tri-valent, $\mathrm{Mg}$ is only known to be bivalent. Our goal is to replace all bivalent $\mathrm{Fe}$ cations induced by the oxygen substoichiometry with $\mathrm{Mg}^{2+}$ cations. The $\mathrm{Mg}^{2+}$ radius is close to the $\mathrm{Fe}^{2+}$ one. A decrease of the leakage current is then expected, associated with the diminution of the $\mathrm{Fe}^{2+}$ content, and the resulting decrease of the $\mathrm{Fe}^{2+} / \mathrm{Fe}^{3+}$ hopping phenomenon.

Films have been elaborated by pulsed laser deposition (PLD) using a $\mathrm{KrF}$ excimer laser, $\lambda=248 \mathrm{~nm}$, with a $5 \mathrm{~Hz}$ repetition rate. The energy density of the laser on the target was tuned to $2.5 \mathrm{~J} / \mathrm{cm}^{2}$ and the distance between the target and the substrate was fixed at $5 \mathrm{~cm}$. A $\mathrm{SrRuO}_{3}(\mathrm{SRO})$ conducting layer was first deposited under $250 \mathrm{mbar}_{2}$ at $655^{\circ} \mathrm{C}$ on $\mathrm{SrTiO}_{3}$ (STO) (111) substrates (Crystec). The substrates were previously cleaned in an ultrasonic cleaner in acetone, isopropanol, distilled water, and ethanol. A GFO:Mg layer of about $200 \mathrm{~nm}$ thickness was then deposited under 200 mbar $\mathrm{O}_{2}$ at $750^{\circ} \mathrm{C}$. After the deposition, the samples were cooled down to room temperature under the processing gas. $\mathrm{Ga}_{0.6} \mathrm{Fe}_{1.4-\mathrm{x}} \mathrm{Mg}_{\mathrm{x}} \mathrm{O}_{3}$ targets used for the elaboration have been formed in a reaction of $\mathrm{Fe}_{2} \mathrm{O}_{3}, \mathrm{Ga}_{2} \mathrm{O}_{3}$, and $\mathrm{MgO}$. The stoichiometric milling was carried out in an attritor mill for one hour in an ammoniacal solution $(p \mathrm{H}=9)$. The solution was then placed in a drying oven until full evaporation of the liquid part. The resulting powder was manually ground. An organic binder (polyvinyl alcohol) has been systematically added at around $3 \mathrm{wt}$. \% to improve the mechanical behavior of the samples. The powders were finally compacted into pellets and sintered in a platinum crucible at $1400^{\circ} \mathrm{C}$ for $20 \mathrm{~h}$ under air. The thin films crystallographic structure was characterized by x-ray diffraction (XRD). $\theta-2 \theta$ scans, $\varphi$-scans, and reciprocal lattice mappings were made using a Rigaku Smart Lab diffractometer equipped with rotating anode having a monochromated copper radiation $\left(K_{\alpha 1}=0.154056 \mathrm{~nm}\right)$. For electric properties measurements, platinum dots top electrodes with a diameter of $0.2 \mathrm{~mm}$ were deposited by magnetron sputtering using a shadow mask onto the GFO films under a pressure of 5 mTorr at room temperature. I-V curves at room temperature were then carried 
TABLE I. EDX analysis, cell parameters, and roughness of the different thin films; the error is given for each in terms of $3 \sigma$.

\begin{tabular}{lcccc}
\hline \hline Expected sample & $\mathrm{Ga} / \mathrm{Fe} / \mathrm{Mg}$ content $^{\mathrm{a}}$ & $\mathrm{a}(\mathrm{nm})$ & $\mathrm{b}(\mathrm{nm})$ & $\mathrm{c}(\mathrm{nm})$ \\
\hline & $0.65(1)$ & & & \\
GFO:Mg $(0 \%)$ & $1.35(1)$ & & & \\
& 0 & $0.862(7)$ & $0.944(3)$ & $0.506(8)$ \\
& $0.65(1)$ & & & \\
GFO:Mg $(0.5 \%)$ & $1.34(1)$ & & & \\
& $0.01(2)$ & $0.868(3)$ & $0.943(2)$ & $0.503(5)$ \\
GFO:Mg $(2.8 \%)$ & $0.62(1)$ & & & \\
& $1.32(1)$ & & & \\
& $0.06(2)$ & $0.874(5)$ & $0.942(2)$ & $0.506(7)$ \\
GFO:Mg $(7.5 \%)$ & $0.60(1)$ & & & \\
\hline
\end{tabular}

${ }^{\mathrm{a}}$ Formulation obtained from EDX measurements.

out using a HP4145B semiconductor parameter analyzer in a voltage range of $[-10 \mathrm{~V}-10 \mathrm{~V}]$.

The chemical compositions of the films were determined from scanning electron microscopy coupled with energy dispersion X-ray analysis (SEM-EDX). The values given in Table I are an average over at least five measurements performed at different locations on the sample, with a standard deviation inferior to $1 \% \mathrm{Wt}$. The magnesium content has been defined by $\mathrm{Mg}(\%)=100 \times[\mathrm{Mg}] /([\mathrm{Mg}]+[\mathrm{Fe}]+[\mathrm{Ga}])$. In agreement with this definition and according to the compositions determination, the samples will be labelled GFO:Mg (0\%), GFO:Mg (0.5\%), GFO:Mg (2.8\%), and GFO:Mg (7.5\%). The XRD measurements confirm that the GFO:Mg films are epitaxially grown on the STO SRO (111) substrates. Figure 1 shows the $\theta-2 \theta$ patterns of the $\mathrm{Mg}$ doped GFO thin films and indicates a $b$-axis oriented epitaxial structure of GFO. Indeed, all $(0 \mathrm{kO})$ expected harmonic

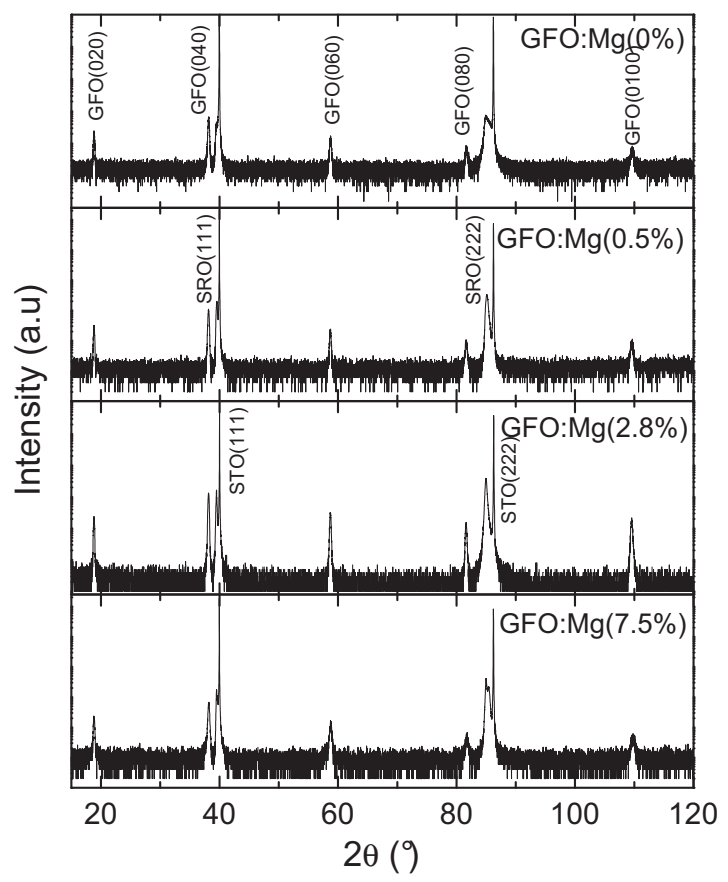

FIG. 1. XRD patterns of the $\mathrm{Mg}$-doped $\mathrm{Ga}_{0.6} \mathrm{Fe}_{1.4} \mathrm{O}_{3}$ thin films.

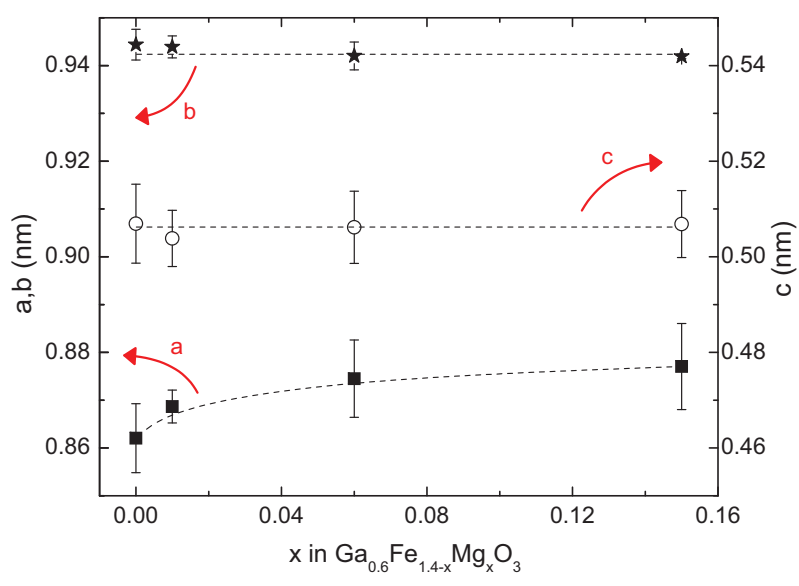

FIG. 2. Evolution of the cell parameters of the $\mathrm{Mg}$-doped $\mathrm{Ga}_{0.6} \mathrm{Fe}_{1.4} \mathrm{O}_{3}$ thin films.

peaks of the GFO compound are observed and no impurities are evidenced. Reciprocal lattice maps of the thin films were performed around reflections $\{062\}$ and $\{570\}$ for all studied compositions. These measurements, combined to the observation of the $\{0 k 0\}$ in $\theta-2 \theta$ mode, allow to determine the lattice parameters. The cell parameters are reported in Table I and plotted in Figure 2. Both $b$ and $c$ cell parameters remain constant whatever the $\mathrm{Mg}$ content. The $a$ cell parameter increases for $\mathrm{Mg}$ contents up to GFO:Mg (2.8\%) and then remains constant. The observed $b$ values are all greater than for the bulk material $\left(b_{\text {bulk }}=9.3993(3) \AA\right) .{ }^{17} \varphi$-scans on the STO and SRO $\{204\}$ reflections and on the GFO:Mg $\{680\}$ reflections have been performed in order to establish the in-plane epitaxial relationships (the measurements performed for the $\mathrm{Ga}_{0.6} \mathrm{Fe}_{1.25} \mathrm{Mg}_{0.15} \mathrm{O}_{3}$ thin film are given as a representative example in Figure 3). All layers (i.e., STO, $\mathrm{SRO}$, and GFO) present six-fold peaks every $60^{\circ}$. This result can be explained considering the following epitaxial relationship: $\mathrm{SrTiO}_{3}$ crystallizes in a cubic structure with $a=3.905 \AA$ and therefore shows a hexagonal pattern along [111]. At room temperature, $\mathrm{SrRuO}_{3}$ crystallizes usually in an orthorhombic structure, which can be described as a slightly distorted pseudo-cubic perovskite cell with a lattice parameter $a=3.92 \AA{ }^{18}$ Consequently, the pattern along

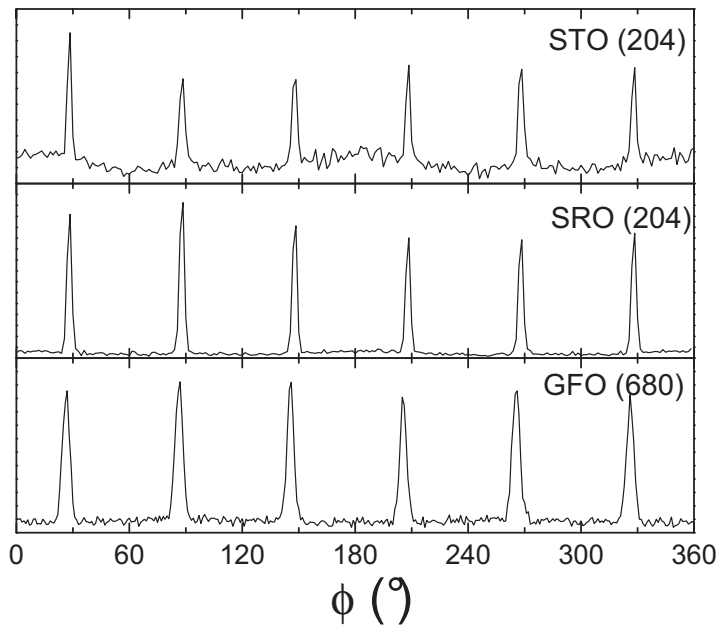

FIG. 3. $\varphi$-scans on the $\mathrm{SrTiO}_{3}$ and $\mathrm{SrRuO}_{3}\{204\}$ reflections and on the $\mathrm{Ga}_{0.6} \mathrm{Fe}_{1.25} \mathrm{Mg}_{0.15} \mathrm{O}_{3}\{680\}$ reflection. 


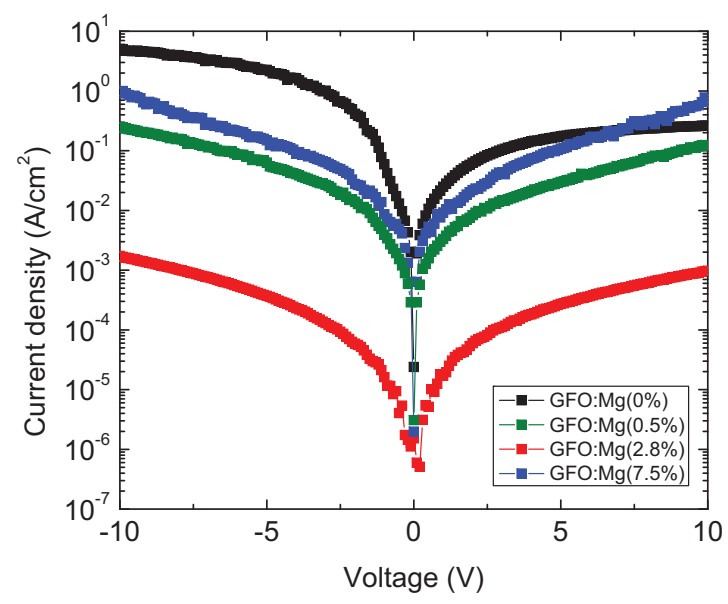

FIG. 4. Variations of leakage current density with the applied voltage at room temperature of the GFO:Mg thin films.

[111] is also a hexagon with an apothem equal to $3.2 \AA$. The mismatch between STO and SRO is less than $1 \%$. The different orientations of the GFO crystallites can be explained considering the different matching possibilities between the GFO and SRO lattices: $c_{\mathrm{GFO}} \approx 5.1 \AA \approx a_{\mathrm{SRO}} \times \sqrt{2}(8 \%$ mismatch) on the one hand and $a_{\mathrm{GFO}} \approx 8.7 \AA \approx 3.2 \times 3(9 \%$ mismatch) on the other hand. The angle between two GFO cells is about $60^{\circ}$ allowing the existence of three variants. Figure 4 shows the variation of the leakage current for the different $\mathrm{Mg}$-doped thin films. The leakage current density for GFO:Mg films is strongly reduced from GFO:Mg $(0.5 \%)$ to GFO:Mg (2.8\%), by roughly four orders of magnitude (Figure 5). For higher $\mathrm{Mg}$ concentration, an increase of the leakage current is observed: increasing the $\mathrm{Mg}$ content from $2.8 \%$ to $7.5 \%$, the leakage current increases by about three orders of magnitude. We attribute the decrease of the leakage currents to the substitution of $\mathrm{Fe}^{2+}$ with $\mathrm{Mg}^{2+}$, decreasing the hopping possibilities between $\mathrm{Fe}^{2+}$ and $\mathrm{Fe}^{3+}$ within the structure. A possible mechanism explaining this behavior can be described using the Kröger-Vink notation. Indeed, the production of $\delta$ oxygen vacancies in $\mathrm{Ga}_{0.6} \mathrm{Fe}_{1.4} \mathrm{O}_{3-\delta}$ during the growth process can be written

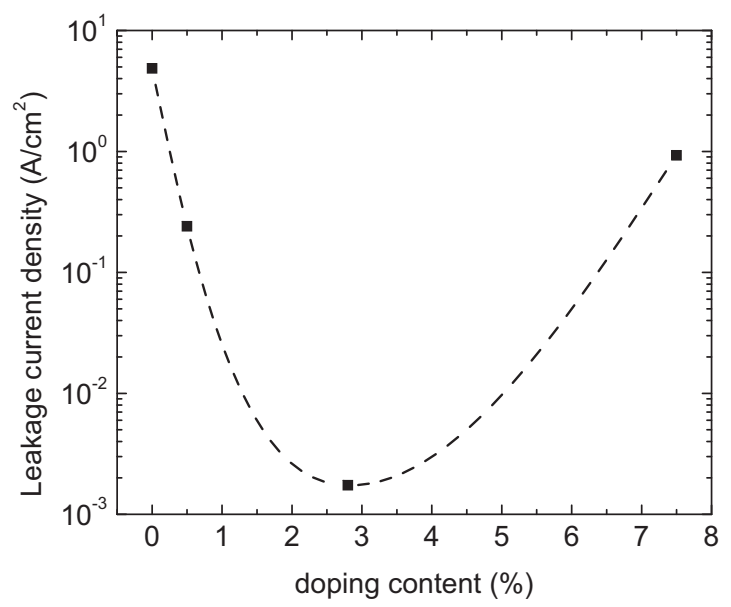

FIG. 5. Variation of the leakage currents density with the increasing doping content in the GFO:Mg thin film.

$$
\delta O_{o}^{\times} \rightarrow \delta\left(V_{o}^{\bullet \bullet}+\frac{1}{2} O_{2}+2 e^{-}\right),
$$

where $V_{o}^{\bullet \bullet}$ is a vacancy in the oxygen site with a double positive charge. $\mathrm{Fe}^{3+}$ acts as an acceptor of electrons and gets reduced into $\mathrm{Fe}^{2+}$

$$
2 \delta e^{-}+2 \delta F e_{F e}^{\times} \Longleftrightarrow 2 \delta F e_{F e}^{\prime} .
$$

The simultaneous presence of $\mathrm{Fe}^{2+}$ and $\mathrm{Fe}^{3+}$ allows electrical conduction via a hopping mechanism.

Substitution of $\mathrm{Fe}$ with $\mathrm{Mg}$ in the $\mathrm{Mg}$-doped GFO thin films leads to the creation of holes

$$
x M g O \rightarrow x\left(M_{F e}^{\prime}+h^{\bullet}+\frac{1}{2} O_{2}\right),
$$

where $M g_{F e}^{\prime}$ stands for $\mathrm{Mg}$ in the Fe site with an apparent negative charge and $h^{\bullet}$ denotes a hole. These holes can be recombined with electrons

$$
e^{-}+h^{\bullet} \rightarrow 0
$$

According to theses notations, the decrease of the leakage currents which occurs until GFO:Mg (2.8\%) can be ascribed to an increasing recombination between holes and electrons. The charge carriers responsible for the leakage currents are the electrons and we have an n-type conduction for $2 \delta>\mathrm{x}$. According to this postulated model and for higher doping contents, the observed behavior might be associated to the increasing presence of holes and we have a p-type conduction for $\mathrm{x}>2 \delta$. This analysis suggests that by controlling the doping atom concentration, it is possible to adjust simultaneously the carriers' density and carriers' type in this thin film material.

In conclusion, we have elaborated $\mathrm{Mg}$-doped GFO thin films by pulsed laser deposition for a magnesium content up to $7.5 \%$ at. X-ray characterizations show a perfect epitaxial growth of the $\mathrm{Mg}$ doped GFO films on the SRO buffered STO(111) substrate with no parasitic phase. We have evidenced an optimum $\mathrm{Mg}$ content of 2.8 at. \% for which the leakage currents undergo a drastic decrease of almost four orders of magnitude. Such a reduction of the leakage currents is extremely promising for enabling future magnetoelectric characterization of the elaborated films. Evolution of the leakage currents might be ascribed to an evolution from n-type to p-type electrical conduction with the increasing doping content. These results open up the possibility to develop a new class of material that exhibits at room temperature non zero magnetization, tunable transport properties, and magnetoelectric properties.

This work was supported by the CNRS PICS program \# 5733 and the Leading Foreign Research Institute Recruitment Program through the National Research Foundation of Korea (NRF) funded by the Ministry of Education, Science and Technology (MEST) (2011-00267).

\footnotetext{
${ }^{1}$ N. Hur, S. Park, P. A. Sharma, J. S. Ahn, S. Guha, and S. W. Cheong, Nature (London) 429, 392 (2004).

${ }^{2}$ Ch. Binek and B. Doudin, J. Phys.: Condens. Matter 17, L39 (2005).

${ }^{3}$ S. Ju, T.-Y. Cai, G.-Y. Guo, and Z.-Y. Li, Phys. Rev. B 75, 064419 (2007).
} 
${ }^{4}$ G. T. Rado, Phys. Rev. Lett. 13, 335 (1964).

${ }^{5}$ T. Arima, D. Higashiyama, Y. Kaneko, J. P. He, T. Goto, S. Miyasaka, T. Kimura, K. Oikawa, T. Kamiyama, R. R. Kumai, and Y. Tokura, Phys. Rev. B 70, 064426 (2004).

${ }^{6}$ M. Trassin, N. Viart, G. Versini, S. Barre, G. Pourroy, J. H. Lee, W. Jo, K. Dumesnil, C. Dufour, and S. Robert, J. Mater. Chem. 19, 8876 (2009).

${ }^{7}$ J. F. Scott, J. Phys.: Condens. Matter 20, 021001 (2008).

${ }^{8}$ G. Lawes and G. Srinivasan; J. Phys. D: Appl. Phys. 44, 243001 (2011).

${ }^{9}$ M. Dawber, K. M. Rabe, and J. F. Scott, Rev. Mod. Phys. 77, 108 (2005).

${ }^{10}$ J. F. Scott, J. Phys.: Condens. Matter 18, R361 (2006).

${ }^{11}$ B. Nagaraj, S. Aggarwal, T. K. Song, T. Sawhney, and R. Ramesh, Phys. Rev. B 59, 16022 (1999).
${ }^{12}$ P. Zubko, D. J. Jung, and J. F. Scott, J. Appl. Phys. 100, 114113 (2006).

${ }^{13}$ H. Hu and S. B. Krupanidhi, J. Mater. Res. 9, 1014 (1994).

${ }^{14}$ H. Liu and Y. Sun, J. Phys. D: Appl. Phys 40, 7530 (2007).

${ }^{15}$ K. H. Yoon, J. C. Lee, J. Park, D. H. Kang, C. M. Song, and Y. G. Seo, Jpn. J. Appl. Phys., Part 1 40, 5497 (2001).

${ }^{16}$ N. Wakiya, Y. Kimura, N. Sakamoto, D. Fu, T. Hara, T. Ishiguro, T. Kiguchi, K. Shinozaki, and H. Suzuki, J. Ceram. Soc. Jpn. 117, 1004 (2009).

${ }^{17}$ S. C. Abrahams, J. M. Reddy, and J. L. Bernstein, J. Chem. Phys. 42(11), 3957 (1965).

${ }^{18}$ C. B. Eom, R. J. Cava, R. M. Fleming, J. M. Philips, R. B. van Dover, J. H. Marshall, J. W. P. Hsu, J. J. Krajewski, and W. F. Peck, Jr., Science 258, 1766 (1992). 